\title{
Gradhiva
}

GRADHIV

Revue d'anthropologie et d'histoire des arts

$14 \mid 2011$

Carl Einstein et les primitivismes

\section{Baptiste Buob, La Dinanderie de Fès. Un artisanat traditionnel dans les temps modernes}

Une anthropologie des techniques par le film et le texte, Paris, Ibis Press-

Éditions de la Maison des sciences de l'homme, 2009

Jean-Charles Depaule

\section{(2) OpenEdition}

Journals

Édition électronique

URL : http://journals.openedition.org/gradhiva/2239

DOI : 10.4000/gradhiva.2239

ISSN : 1760-849X

Éditeur

Musée du quai Branly Jacques Chirac

Édition imprimée

Date de publication : 30 novembre 2011

Pagination : 260-261

ISBN : 978-2-35744-046-3

ISSN : 0764-8928

Référence électronique

Jean-Charles Depaule, "Baptiste Buob, La Dinanderie de Fès. Un artisanat traditionnel dans les temps modernes », Gradhiva [En ligne], 14 | 2011, mis en ligne le 30 novembre 2011, consulté le 21 septembre 2020. URL : http://journals.openedition.org/gradhiva/2239; DOI : https://doi.org/10.4000/gradhiva. 2239

Ce document a été généré automatiquement le 21 septembre 2020.

(C) musée du quai Branly 


\section{Baptiste Buob, La Dinanderie de Fès. Un artisanat traditionnel dans les temps modernes}

Une anthropologie des techniques par le film et le texte, Paris, Ibis PressÉditions de la Maison des sciences de l'homme, 2009

Jean-Charles Depaule

\section{RÉFÉRENCE}

Baptiste Buob, La Dinanderie de Fès. Un artisanat traditionnel dans les temps modernes. Une anthropologie des techniques par le film et le texte, Paris, Ibis Press-Éditions de la Maison des sciences de l'homme, 2009, 420 p. +30 pl., 2 rabats, et 1 DVD 
1 Baptiste Buob le rappelle d'entrée de jeu, la représentation dominante, et tenace, de l'artisanat marocain est fortement associée à l'image d'une personnalité culturelle qui, avec ses savoirs ancestraux, se serait fidèlement maintenue à travers les siècles.

Dès le XIII ${ }^{\mathrm{e}}$ siècle, le cuivre jaune ou laiton, extrait et fondu localement, notamment à Fès, était exporté sous forme de lingots vers «le pays des Noirs» et vers la Méditerranée musulmane. À Fès, il était aussi transformé et les objets qui y étaient produits également exportés. Quelquesuns, parvenus jusqu'à nous, témoignent d'un art ancien influencé par des savoirfaire berbères, ou originaires de l'Orient arabe, de l'Afrique noire et de l'Andalousie. Mais ce que l'on nomme

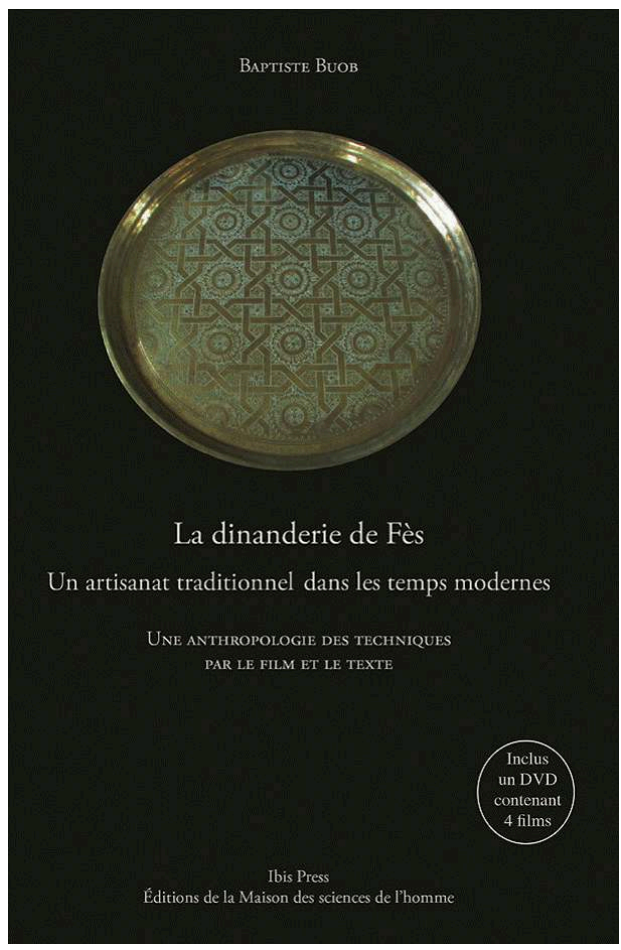
aujourd'hui « artisanat traditionnel » est le résultat d'une construction. « $\mathrm{La}$ dinanderie, écrit Baptiste Buob, n'est pas une tradition ancestrale, elle est devenue traditionnelle. » (p. 102) En effet, depuis le début du $\mathrm{xx}^{\mathrm{e}}$ siècle, elle a évolué selon deux tendances divergentes: d'une part produire des objets de luxe, dans le prolongement de l'action du protectorat français (1912-1956) qui privilégiait ses aspects « artistiques »; d'autre part fabriquer des objets d'un usage plus courant, souvent en relation avec la consommation du thé. Ce développement a eu pour conséquence une différenciation des types de division du travail, des qualifications et des modes de transmission des savoirs, une diversification des lieux de production - ateliers, anciens caravansérails ou fabriques, qui se trouvent en majorité dans la médina - et des réseaux de commercialisation.

3 Parmi les principaux facteurs de transformation, Baptiste Buob insiste sur la déstructuration, largement imputable au protectorat, de la hisba, vieille institution urbaine du monde musulman contrôlant l'organisation et l'exercice des métiers. Et sur la disparition, après l'indépendance, des artisans juifs détenteurs des savoir-faire d'un artisanat situé assez bas dans l'échelle des métiers : travailler les métaux « auréolés de croyances occultes » leur était réservé, ainsi qu'à "des classes sociales méprisées » (p. 42).

4 Autre facteur majeur: l'introduction au XVIII ${ }^{\mathrm{e}}$ siècle, par les Anglais, du thé dont la consommation s'est progressivement généralisée, avec l'emploi d'accessoires métalliques spécifiques, théières, plateaux, samovars, sucriers... Afin de contrecarrer la concurrence de l'argenterie d'importation, connue sous l'appellation rayt, arabisation de Wright, nom d'un industriel de Manchester associé à des commerçants marocains établis dans cette ville, on produit à Fès, entre les deux guerres, de plus en plus de ces " objets du thé » s'en inspirant, en cuivre argenté ou brut - le brut ayant de façon générale la préférence des touristes. Parmi eux il y a les plateaux ovales (ovâl dans le parler des dinandiers) munis de pieds, fondus, comme d'autres pièces de garniture, 
dans la médina, et bordés par une ceinture soudée dans laquelle sont ménagées des anses. De la découpe des fonds à l'argenture, Baptiste Buob détaille les treize phases de leur fabrication, à laquelle est consacré le premier des quatre films joints au livre (tandis que le deuxième traite de la fonderie). Il analyse la révolution technique induite par l'usage du thé : adoption de formes exogènes, de matières nouvelles comme le maillechort, recours à la soudure ainsi qu'à l'argenture par électrolyse, emploi de machines (tours, laminoirs et polissoirs).

5 Le poids croissant, dans le fonctionnement économique et social de la dinanderie, des "maîtres à la sacoche " ou "maîtres à l'argent", capitalistes marchands qui, à la différence des « maîtres avec les mains » ou " maîtres au marteau », ne " mettent pas la main à la pâte » (p.157), a contribué à précipiter le changement au cours des dernières décennies.

6 La confection des ovâl appartient au secteur « ordinaire » de la dinanderie fassie, où la condition des travailleurs, aujourd'hui d'origine surtout rurale, est précaire, et où, main-d'œuvre docile, les enfants sont nombreux (on notera aussi, à certaines phases, l'intervention de femmes). L'atomisation du travail et de ses localisations y est extrême, et, au cours de sa fabrication, un même article se déplace selon un complexe maillage. La réalité présente contredit donc cet autre stéréotype : l'idée d'un artisan travaillant seul, ou presque, dans son échoppe où il accomplirait l'ensemble de la chaîne opératoire.

7 La gravure des grands plateaux circulaires, ou tîfor, destinés à une clientèle aisée, et leur mise en forme finale par un « dresseur ", qui donne son volume à la plaque gravée, appartiennent à la branche "noble » de la dinanderie actuelle. Le travail y est moins répétitif, moins fractionné et éclaté dans l'espace, la qualification des artisans, leur attachement au métier et leur implication personnelle y sont nettement plus élevés. Schémas à l'appui, Baptiste Buob explicite la géométrie des canevas qui guident le graveur et la saisit « en mouvement » dans le troisième film de la série.

D'eux-mêmes les dinandiers disent qu'ils sont « tantôt torchon, tantôt lampion ». Selon le secteur où ils travaillent, ils sont diversement soumis aux aléas de l'embauche, sont considérés ou dépréciés en fonction de la qualification qui est exigée d'eux et leur est reconnue. Baptiste Buob décrit la manière dont ils se perçoivent et leur façon de combattre la "sauvagerie » ou de conjurer le glissement progressif vers l'anonymat ouvrier - dénégation, mise à distance, survalorisation (nostalgique) ou révolte.

9 Leur univers, où l'apprentissage "peut s'apparenter à une véritable forme de dressage" (p. 290), est soumis à des rapports hiérarchiques rigides, lisibles dans l'espace de travail : le m'âllem, le «maître ", demeure immobile à son poste tandis que les apprentis ou les enfants qui n'ont pas de place fixe assurent la circulation des pièces aux différents stades de leur transformation. L'auteur note en outre: «La hiérarchie spatiale des corps ne s'exprime pas uniquement sur le plan horizontal, mais plus encore sur le plan vertical. Les enfants travaillent généralement accroupis, jamais à hauteur d'un maître ou d'un ouvrier. » (p. 294)

10 Le noyau central du livre est constitué par l'enquête proprement dite sur les chaînes opératoires, les savoir-faire, les matières, les outils et leur maniement, les machines, les gestes et les postures, les positions et les mouvements, les logiques décoratives. Son ethnographie rigoureuse de l'univers changeant de la dinanderie fassie nourrit une précieuse anthropologie des techniques ${ }^{1}$. 
11 Une anthropologie par le film et le texte, précise le sous-titre de l'ouvrage. L'approche cinématographique restitue une topographie de la dinanderie qui n'est pas réductible à la description textuelle ni aux cartes et diagrammes que l'auteur a établis systématiquement. C'est celle des corps, de leur interaction et de la manière dont le travail qu'ils effectuent prend place dans l'atelier ou l'échoppe et dans l'espace de la médina ; des objets et des individus circulant d'un lieu à l'autre; des rythmes sonores (cadences de la percussion des outils, ponctuation des voix) et visuels (durée et réitération des tâches, amplitude des gestes répétés...), que le film "sauve ». On ne saurait donc trop conseiller au lecteur de ne pas laisser longtemps de côté ceux qui sont insérés dans l'ouvrage, les trois déjà évoqués et le quatrième, qui suit, au travail et à d'autres moments de sa journée, un graveur installé dans une boutique-atelier, place Seffarine (place des Dinandiers) - le choix dans ce cas étant moins de s'attacher à un objet que « de faire de l'artisan le protagoniste » (p. 383).

Baptiste Buob revient dans une annexe sur sa démarche filmique, sur son apport spécifique $^{2}$. Il revient notamment sur l'intérêt de décrire non seulement par le texte mais par l'image l'enchaînement des gestes techniques et des tâches, des postures, l'engagement des corps, en ne retenant pas les seuls points saillants ou temps forts, et de rendre compte des continuités et, le mieux possible, de l'enchevêtrement des processus. Il insiste également sur la mise en scène de soi qu'un individu propose (impose, oppose) au cinéaste, comme on le voit dans «la théâtralisation de la soumission " qui se manifeste dans cet univers très hiérarchisé. Et ce sur quoi, en revanche, le regard ne se focalise pas, l'image filmée captant toujours plus que ce qu'elle est censée décrire. Par exemple, le décor des ateliers, juste évoqué dans le texte : photographies, affiches, objets prophylactiques, diplômes... fixés au mur. Il s'agit de ce que l'on voit sans véritablement le voir, et de ce que l'on pourrait entendre si on y prêtait attention: non seulement les cadences mais la qualité des sons, comme celui, mat, des paumes et celui des poings fermés qui tassent alternativement le sable des moules utilisés dans la fonderie. D’un film à l'autre, on est frappé aussi par le rôle joué par la voix, parcimonieuse dans "Ovâl», abondante dans "Place Seffarine», où l'artisan négocie avec l'herboriste pour lequel il grave une enseigne, et, plus largement, par les environnements sonores et donc les bruits hors champ. Notant que l'ouie « est sans doute l'un des sens les plus mobilisés dans ces activités ", Baptiste Buob suggère que d'autres chercheurs puissent exploiter cette dimension de ses films, sur laquelle il ne s'est pas concentré (p. 388).

Son ouvrage contribue de façon magistrale à l'anthropologie des techniques. Il est en outre une superbe illustration de l'intelligibilité (sensible) que « l'anthropologie par le film» est capable d'apporter. Souhaitons qu'il contribue à lever le soupçon qui continue trop souvent de peser sur elle. 


\section{NOTES}

1. Un regret: ne pas disposer d'un index et/ou d'un lexique qui rende plus accessibles encore la collecte et l'analyse des technolectes, et plus largement du vocabulaire du monde de la dinanderie de Fès.

2. Dans cette réflexion méthodologique, Baptiste Buob «colle " assez étroitement aux notions élaborées par Claudine de France. Je comprends la portée de l'hommage ainsi rendu, tout en me demandant si cela ne risque pas de gommer quelque peu les vertus propres de son travail, qui n'est pas qu'un exercice d'application très réussi.

\section{AUTEURS}

\section{JEAN-CHARLES DEPAULE}

jean-charles.depaule898@orange.fr 